\title{
PNA-Dependent Gene Chemistry: Stable Coupling of Peptides and Oligonucleotides to Plasmid DNA
}

BioTechniques 28:304-316 (February 2000)

O. Zelphati, X. Liang, C. Nguyen, S. Barlow', S. Sheng ${ }^{2}$, Z. Shao ${ }^{2}$ and P.L. Felgner Gene Therapy Systems, San Diego, CA, ${ }^{1}$ San Diego State University, San Diego, CA, and ${ }^{2}$ University of Virginia, Charlottesville, VA, USA

\section{ABSTRACT}

Two approaches are described for stably conjugating peptides, proteins and oligonucleotides onto plasmid DNA. Both methods use a peptide nucleic acid (PNA) clamp, which binds irreversibly and specifically to a binding site cloned into the plasmid. The first approach uses a biotin-conjugated PNA clamp that can be used to introduce functional biotin groups onto the plasmid to which streptavidin can bind. Atomic force microscopy images of linearized plasmid show streptavidin localized at the predicted PNA binding site on the DNA strand. Peptides and oligonucleotides containing free thiol groups were conjugated to maleimide streptavidin, and these streptavidin conjugates were bound to the biotin-PNA-labeled plasmid. In this way, peptides and oligonucleotides could be brought into stable association with the plasmid. A second approach used a maleimide-conjugated PNA clamp. Methods are described for conjugating thiolated peptides and oligonucleotides directly to the maleimide-PNA-DNA hybrid. This straightforward technology offers an easy approach to introduce functional groups onto plasmid DNA without disturbing its transcriptional activity.

\section{INTRODUCTION}

Plasmid-based, nonviral gene delivery systems offer a promising therapeutic approach for the treatment of acquired diseases, infectious diseases and inherited disorders $(5,11,12,15)$. However, their efficiencies and clinical potencies are limited today by low-level gene product expression (10). The most commonly explored approach for increasing in vivo plasmid expression is to develop improved delivery systems by mimicking the advantageous aspects of viral systems with simplified components. The key features for such nonviral delivery systems are (i) DNA condensation, (ii) cell surface targeting, (iii) endosomal release, (iv) nuclear uptake and (v) avoidance of nonspecific absorption of the delivery system by nontarget tissues.

The delivery systems under investigation today are designed to dissociate from the plasmid after intracellular delivery because the release of DNA from the carrier is usually considered a requirement for transcription to begin $(20,21,23)$. However, for some purposes such as improved nuclear uptake, better cytoplasmic delivery, cell-specific targeting or DNA immunization, it would be desirable to make permanent, irreversible modifications to the plasmid. Unfortunately, there are no wellestablished methods for making irreversible modifications to plasmid in a manner that does not interfere with its transcriptional activity. The peptide nucleic acid (PNA)-dependent gene chemistry (PDGC) technology de- scribed in this report was developed to provide a way to make permanent modifications to plasmids without interfering with their transcriptional activity.

PNA is a polynucleic acid analog that has the deoxyribose-phosphate backbone replaced by a peptide backbone $(4,6,17)$. PNA can hybridize with complementary DNA in a typical Watson/Crick manner except that the affinity of the PNA-DNA hybrid is much stronger than the DNA-DNA hybrid. This is because of the neutral charge of the PNA backbone, which does not introduce a repulsive force to drive the hybridized strands apart $(2,4,7,8,17)$. PNA has been shown capable of invading duplex DNA and hybridizing to plasmid to form a stable PNA-DNAPNA triplex hybrid, while displacing the native complementary DNA strand to form a nonhybridizing D-loop $(2,3)$.

We previously reported that fluorescein- or rhodamine-conjugated PNA clamps could be hybridized to supercoiled DNA, which leads to the production of a highly fluorescent plasmid and which could be used to follow the delivery of the DNA in living cells (22). A PNA clamp is an oligomer that has two identical PNA sequences joined by a flexible hairpin linker $(8,13)$. Binding of the PNA clamp to its DNA target is sequence-specific, stoichiometric, extremely stable and essentially irreversible under biological conditions $(8,22)$. The use of rhodamine-labeled, plasmid-encoding green fluorescent protein (GFP) provided a dual fluorescent system for rapid, quantitative and simul- 
taneous monitoring of both plasmid biodistribution and gene expression in real time (22). In this report, we extend these findings to show that PNA clamps containing either biotin or maleimide can be used to synthesize plasmids containing peptides and oligonucleotides.

\section{MATERIALS AND METHODS}

\section{PNA, Oligonucleotide, Plasmid DNA and Peptide}

The PNA used for these studies was designed as a clamp as previously reported $(8,22)$. PNA-biotin, -SMCC [succinimidyl-4-( $N$-maleimidomethyl) cyclohexane-1-carboxylate], -rhodamine and -fluorescein were purchased from PE Biosystems (Foster City, CA, USA). The PNA sequences used were 5'-TCTCTCTC-O-O-O-JTJTTJTJT-3' (PNA1) and 5'-TTCCTTCC-O-O-OJJTTJJTT-3' (PNA2) (22). Biotin, SMCC, rhodamine and fluorescein are covalently attached to the PNA at the $5^{\prime}$ end. The $3^{\prime}$ thiol modified $\mathrm{CpG}$ oligonucleotide (CpG ODN; 5'-Fl- TGACTGTGAACGTTCGAGATGA- $\left(\mathrm{CH}_{2}\right)_{3}$ $\left.\mathrm{S}-\mathrm{S}-\left(\mathrm{CH}_{2}\right)_{3}-\mathrm{OH}-3^{\prime}\right)$ was purchased from Sigma-Genosys (The Woodlands, TX, USA). The ODN were labeled with fluorescein at their $5^{\prime}$ end. Plasmid DNA (Figure 1) was derived from a high-expression vector based on the human cytomegalovirus (hCMV) immediate early gene promoter/enhancer plus intron $\mathrm{A}$ and a super transcription terminator (pGeneGrip ${ }^{\mathrm{TM}}$; Gene Therapy Systems, San Diego, CA, USA). The pGeneGrip blank or $\beta$-galactosidase plasmid containing a single PNA binding site was constructed as reported (22). For the construction of plasmid containing multiple PNA binding regions, two complementary oligonucleotides were synthesized and annealed to form a $101 \mathrm{bp}$ DNA fragment containing 10 PNA binding sites (16) and a $5^{\prime}$ overhang with sequence GATC at the ends for inserting into the BamHI or $B g l$ II cloning site. The pGeneGrip plasmid containing two binding regions was constructed by inserting the $101 \mathrm{bp}$ fragment into a $B g l \mathrm{II}$ site of the original pGeneGrip vector (Figure 4A). The nuclear localization signal peptide from simian virus 40 (SV40) T-antigen ho- mologue was purchased from Sigma (St. Louis, MO, USA).

\section{Preparation of DNA/PNA Conjugates}

DNA/PNA-biotin-SMCC, -rhodamine or -fluorescein. The PNA clamp was mixed with the plasmid (pDNA, gWIZ $^{\mathrm{TM}}$ vectors; Gene Therapy Systems) as described (16). After the incubation period, free PNA conjugates were completely removed by ethanol precipitation, and the dried plasmid pellet was resuspended in sterile filtered Hepes-buffered saline (HBS; 10 mmol/L Hepes, $50 \mathrm{mmol} / \mathrm{L} \mathrm{NaCl}, \mathrm{pH}$ 7.2) overnight at $4^{\circ} \mathrm{C}$. Plasmid recovery was determined spectrophotometrically at $260 \mathrm{~nm}$. Removal of free PNA was confirmed by Sephacryl-500HR (Amersham Pharmacia Biotech, Piscataway, NJ, USA) gel filtration column.

DNA/PNA-Biotin -StreptavidinFITC or Streptavidin-Gold. The 10 nm diameter Colloidal Gold-labeled streptavidin or streptavidin-FITC (Sigma) was mixed with biotin-PNA-labeled plasmid at 10:1 molar ratio excess and incubated for $1 \mathrm{~h}$ at $37^{\circ} \mathrm{C}$. After the incubation, the mixture was passed over a Sephacryl-500-HR column to remove the free streptavidingold or -FITC.

\section{Coupling of NLS Peptide and ODN to Streptavidin-SMCC}

First, the nuclear localization signal (NLS) peptide was fluorescently labeled using Alexa ${ }^{\mathrm{TM}} 488$ Protein Labeling Kit (Molecular Probes, Eugene, OR, USA). The labeled NLS-peptide was subsequently reduced for $2 \mathrm{~h}$ with 10 molar excess of tris-(2-carboxyethyl) phosphine, hydrochloride (TCEP; Molecular Probes). After the peptide reduction, an equal molar amount of streptavidin-SMCC (Sigma), was added to the NLS-peptide and incubated at $37^{\circ} \mathrm{C}$ water bath for $1 \mathrm{~h}$. The uncoupled peptide was removed by Sephadex G50 column chromatography. Fluorescence of each fraction was measured in a dual scanning fluorometer (FluoroMax-2 ${ }^{\circledR}$; Instruments S.A., Edison, NJ, USA). The Alexa labeled NLS-peptide coupled to streptavidinSMCC was recovered and used to bind the biotin-PNA-labeled plasmid.
Fluorescent $\mathrm{CpG}$ oligonucleotide dissolved in PBS at $2 \mathrm{mg} / \mathrm{mL}$ was reduced with $40 \mathrm{mmol} / \mathrm{L}$ of dithiothreitol (DTT; Life Technologies, Gaithersburg, MD, USA) for $18 \mathrm{~h}$ at room temperature and then purified by Sephadex G-50 (Amersham Pharmacia Biotech) gel filtration column. Absorbance at $260 \mathrm{~nm}$ of the collected fraction was determined, and fractions containing the CpG ODN were pooled. The reduced and purified $\mathrm{CpG}$ ODN was then mixed with streptavidin-SMCC at 5:1 molar ratio of $\mathrm{CpG} \mathrm{ODN}$ to streptavidinSMCC and let stand at room temperature for at least $4 \mathrm{~h}$. The CpG ODNstreptavidin conjugate was subsequently bound to biotin-PNA/DNA and visualized after gel electrophoresis under UV.

\section{Cryo-Atomic Force Microscopy (AFM) (19)}

Streptavidin-FITC attached to BamHI linearized pDNA/DNA-biotin sample was concentrated using Centricon-100 ${ }^{\mathrm{TM}}$ (Millipore, Bedford, MA, USA). The PBS buffer was exchanged for TE buffer $(10 \mathrm{mmol} / \mathrm{L}$ Tris- $\mathrm{HCl}, 1$ mmol/L EDTA, pH 8.0). For AFM, the DNA sample was diluted to $2 \mu \mathrm{g} / \mathrm{mL}$, adsorbed to a $5 \times 5 \mathrm{~mm}$ square cleaved mica wafer (SPI Supplies, West Chester, PA, USA) coated with $1 \mathrm{mmol} / \mathrm{L}$ spermidine (Sigma) and let stand at room temperature for $5 \mathrm{~min}$. The mica was then washed extensively with TE buffer and briefly rinsed with deionized water. The sample was dried under nitrogen and then cooled to liquid nitrogen temperature. The scope was operated at 80 $\mathrm{K}\left(-190^{\circ} \mathrm{C}\right)$. The data was collected in the contact mode at a scanning rate of 1-2 Hz. The images were only flattened and no other manipulation was used.

\section{In-Vitro Transfection and Transmis- sion Electron Microscopy (TEM)}

We plated 200000 COS-1 cells onto Thermanox ${ }^{\mathrm{TM}}$ coverslips (Nalge Nunc International, Rochester, NY, USA) placed inside a 6 -well plate, $24 \mathrm{~h}$ before transfection. Five micrograms of blank mammalian plasmid DNA vector (gWIZ; Gene Therapy Systems) were used per well. DNA/PNA-biotin-streptavidin-gold was mixed with DMRIE/ DOPE (50:50) as described (16). The 
cell transfection was done as previously reported (9). After $48 \mathrm{~h}$ of transfection, the transfected cells were rinsed with $1 \times$ PBS, fixed with $3.3 \%$ formaldehyde, $1 \%$ glutaraldehyde in PBS and stored at $4^{\circ} \mathrm{C}$. The samples were post-fixed in $2 \%$ osmium tetroxide in distilled water and dehydrated in an alcohol series. Then, the samples were embedded in EponEMBed 812 (Electron Microscopy Sciences, Fort Washington, PA, USA), trimmed and mounted on a $\mathrm{LKB}^{\mathrm{TM}}$ Nova ultramicrotome. Approximately $90 \mathrm{~nm}$ thin sections were cut using a Diatome diamond knife (Electron Microscopy Sciences). The sections were picked up on formvar-coated grids. The sections were viewed on a Philips 410 TEM either unstained or stained with uranyl acetate and lead citrate. Finally, the images were captured on Kodak SO-163 film (Eastman Kodak, Rochester, NY, USA).

\section{Coupling of NLS Peptide and ODN to DNA by PNA-SMCC}

We reduced $1 \mathrm{mg}$ of NLS-peptide dissolved in $200 \mu \mathrm{L}$ of $\mathrm{Na}$ phosphate buffer with $5 \mathrm{mmol} / \mathrm{L}$ of TCEP for $2 \mathrm{~h}$ at room temperature. After reduction, DNA/PNA-SMCC and reduced NLS peptide were mixed at 20 moles of NLS peptide/mole of PNA-SMCC in a coupling reaction buffer $(100 \mathrm{mmol} / \mathrm{L} \mathrm{Na}$ phosphate, $150 \mathrm{mmol} / \mathrm{L} \mathrm{NaCl}, \mathrm{pH} 7.2$ ) and incubated at least $4 \mathrm{~h}$ at room temperature. The DNA/PNA-SMCC-peptide and the uncoupled peptide were separated by isopropanol precipitation. The dried pellet was dissolved in deionized water and the amount of pDNA recovered was measured by absorbance at 260 $\mathrm{nm}$. Finally, the reaction product was analyzed by agarose gel electrophoresis.

For the coupling of the fluorescent CpG ODN to DNA/PNA-SMCC, the ODN was first reduced as described

Figure 1. Principal of PDGC. PNA, which is a polynucleic acid analog that has the deoxyribosephosphate backbone of DNA replaced by a peptide backbone, is the core of the PDGC technology. The PNA clamp hybridizes with its complementary binding site on a plasmid to form a highly stable PNA-DNA-PNA triplex clamp. The location of the PNA binding site on the plasmid construct is shown along with the different PNA clamps that can be used. above. The CpG ODN was then mixed with DNA/PNA-SMCC at 20:1 molar ratio for at least $4 \mathrm{~h}$ at room temperature. The DNA/PNA-SMCC-CpG ODN conjugate was subsequently purified by isopropanol purification and visualized by agarose gel electrophoresis.

\section{Gel Electrophoresis Analysis}

We analyzed $2 \mu \mathrm{g}$ of DNA/PNA or DNA/PNA-peptide or -ODN conjugates on a $0.8 \%$ TAE agarose gel and electrophoresed them for $2 \mathrm{~h}$ at $82 \mathrm{~V}$. To visualize the fluorescently labeled

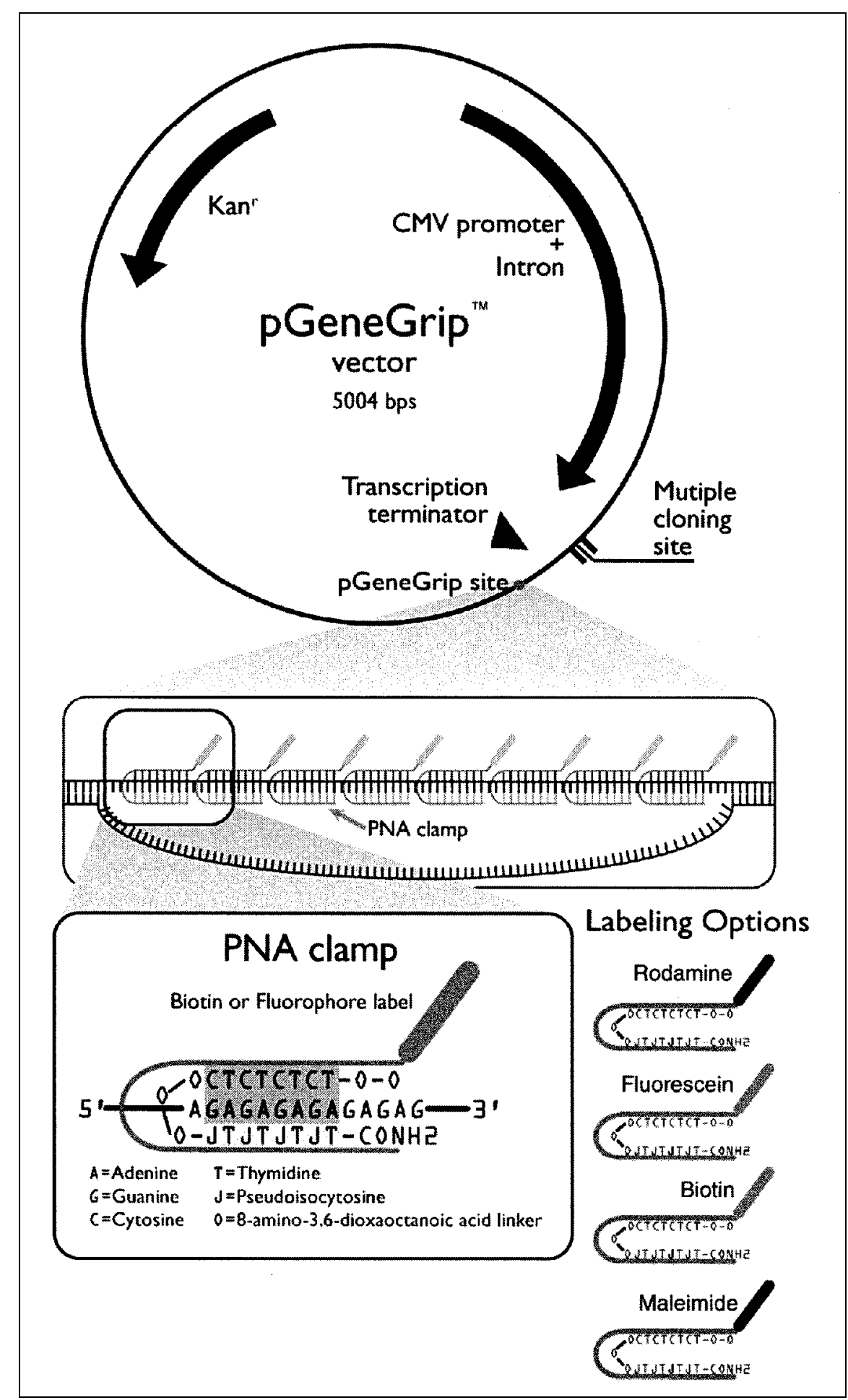


peptide or ODN attached to the DNA/PNA, it is critical to use an ethidium bromide-free gel box. For the direct coupling of the NLS peptide to DNA/PNA-SMCC, the peptide was labeled after coupling by using the ATTO-TAG labeling kit ${ }^{\mathrm{TM}}$ (Molecular Probes). Then, the fluorescence from the peptide or ODN attached to pDNA was visualized and photographed with the UV-transilluminator Model TM10E (UVP, Upland, CA, USA). After visualization of the fluorescent peptide or ODN, gels were labeled with ethidium bromide to check DNA integrity. The photographs were scanned using a HP ScanJet 2CX/T ${ }^{\mathrm{TM}}$ (Hewlett Packard) before and after staining the gel with ethidium bromide.

\section{RESULTS}

\section{Principal of PDGC Technology}

PNA-labeled plasmids were prepared as shown in Figure 1. An 80 bp polypurine AG repeat sequence (PNA Binding Site 1, PNABS1) was cloned after the terminator of a CMV immediate early gene promoter-based plasmid. This region of the plasmid was selected for insertion of the binding site because it is not involved in transcription and PNA binding to this region does not affect expression (22). A complementary PNA clamp molecule was synthesized consisting of an 8-base CT repeat, a 3-unit flexible linker (8-amino-3, 6, -dioxaoctanoic acid), and an 8-base JT repeat ( $\mathrm{J}$ is pseudoisocytosine, an ana$\log$ of $\mathrm{C}$, which encourages formation of the Hoogsteen triplex hybrid) $(8,22)$. The CT stretch hybridizes to the AG repeat on the plasmid in an antiparallel Watson-Crick manner and the JT stretch binds in the major groove of the PNA-DNA hybrid by Hoogsteen interactions to form the PNA-DNA-PNA triplex clamp (8). The nontarget DNA strand is displaced forming the nonhybridized D-loop $(2,3)$.

To further illustrate the specificity and selectivity of PNA clamp binding, a second PNA binding site (PNABS2) with the repeating sequence AAGG was cloned into the pGeneGrip plasmid. This site was created on a $101 \mathrm{bp}$ synthetic duplex oligonucleotide with sticky ends, and the oligonucleotide was cloned into a restriction site as shown on the pGeneGrip plasmid map in Figure 2. A complementary PNA clamp molecule was synthesized consisting of an 8-base CCTT repeat on the Watson-Crick portion of the clamp and an 8-base JJTT repeat on the Hoogsteen portion. The two fluorescently labeled PNA clamps were added to the plasmid, the plasmid was restricted and the fluorescence of the restriction fragments was examined on agarose gels. The results in Figure 2 show that the CT PNA clamp (PNA1) binds only to PNABS1 and the CCTT PNA clamp (PNA2) binds only to PNABS2. In addition to showing the specificity and selectivity of PNA clamp binding, these results illustrate that different molecular species can be specifically attached to the same plasmid by binding different PNA clamps selectively to different sites on the plasmid. We have also shown that the number of PNA binding sites can be systematically increased in plasmids by increasing the number of oligonucleotides cloned into the plasmid. In this way, eight of the $101 \mathrm{bp}$ oligonucleotides were cloned into a single plasmid, resulting in a plasmid that contained 80 potential PNA clamp binding sites (data not shown).

\section{Use of the Biotin-Streptavidin Approach to Attach Ligands to Plasmid DNA}

One approach uses the biotin-streptavidin system for coupling peptides, proteins, oligonucleotides or other types of ligands to DNA. Here, a DNAPNA-biotin hybrid is used to capture streptavidin. A number of straightforward chemical methods are available for covalently attaching peptides and proteins to streptavidin. For example, any ligand that contains a free sulfhydryl group will react with streptavidin that contains a conjugated maleimide moiety. Peptide-streptavidin conjugates can be added directly to biotin-PNA-DNA leading to the generation of a product of the kind shown schematically in Figure 4.

To demonstrate the feasibility of this approach, we prepared streptavidin-labeled plasmid DNA as shown in the Figure 3A. First, biotin-PNA was added 
to the plasmid and the unbound biotinPNA was removed by ethanol precipitation. Streptavidin was added to the biotin-PNA-labeled plasmid, and this product was purified by gel filtration to remove unbound streptavidin. Quantitative analysis of the gel filtration data showed that there was approximately 1 bound streptavidin for every plasmid (data not shown). After streptavidin labeling was carried out as described above and illustrated in Figure 3A, the plasmid that contains a single Bam $\mathrm{H} 1$ site 310 bp away from the PNA binding site was restricted with the BamH1 enzyme. Cryo-AFM images of the streptavidin-labeled DNA are shown in Figure 3B. The micrographs revealed linearized DNA and a white dot on each strand that showed the location of the streptavidin molecule. Virtually every strand had a single streptavidin posi- tioned precisely 310 bp away from the end of each strand at the predicted location of the PNA binding site.

These results illustrate the exquisite specificity and selectivity of this approach for labeling plasmid DNA. The results in Figure 3C show that streptavidin-gold-labeled plasmid can be produced in this way and that intracellular plasmid can be localized by transmission electron microscopy on transfect-

A
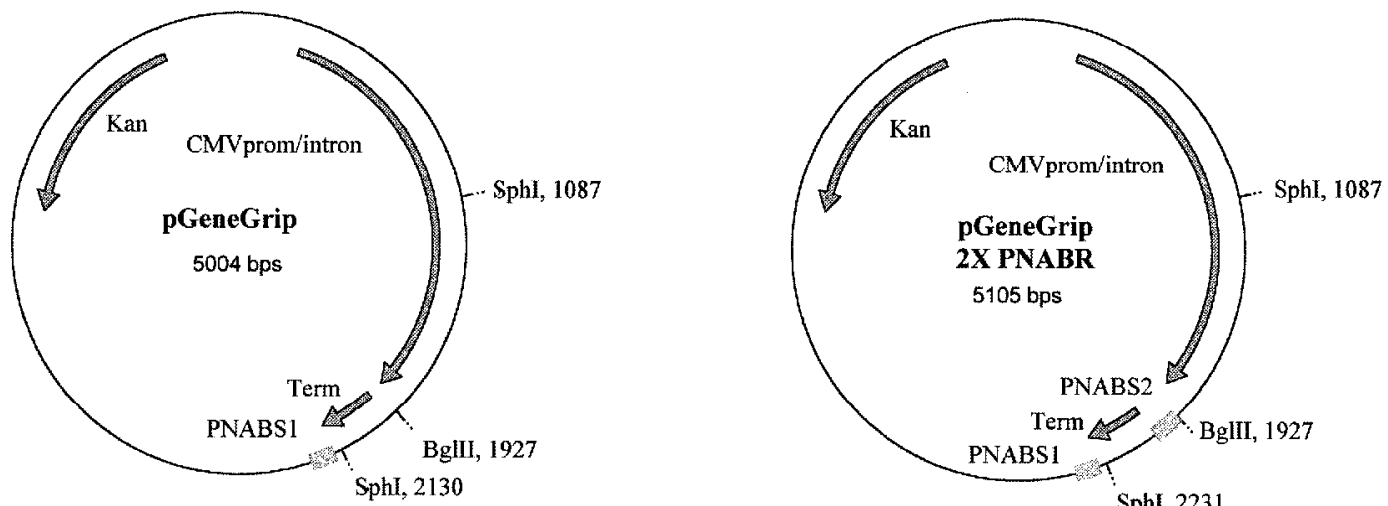

B

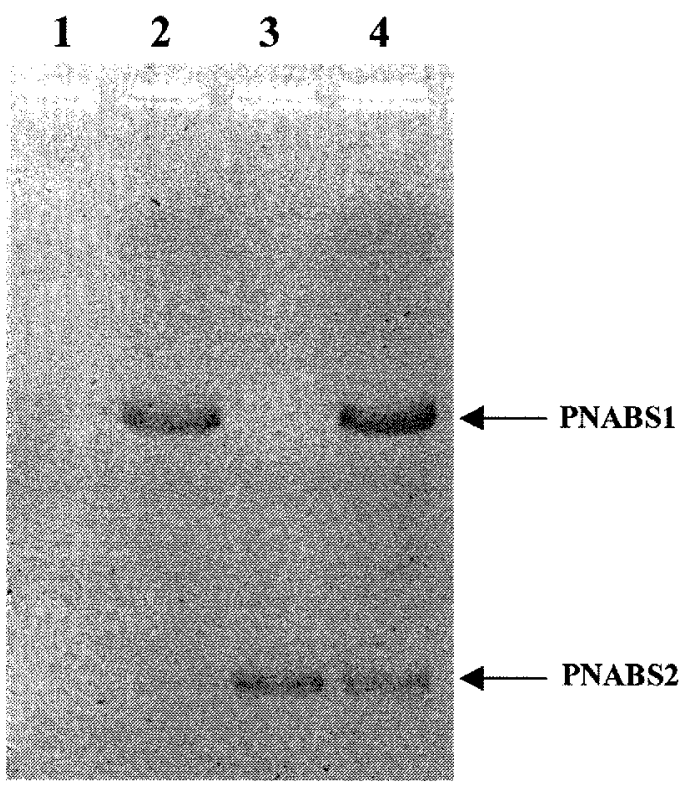

Figure 2. Binding of fluorescent PNA to a plasmid bearing multiple binding regions. (A) Maps of pGeneGrip plasmids containing one or two different types of PNA binding sites (PNABS1 and PNABS2). (B) Agarose gel electrophoresis of SphI-restricted pGeneGrip 2× PNABS plasmid (with PNABS1 and PNABS2). The DNA was visualized under UV illumination by the fluorescence from fluorescein-PNA or rhodamine-PNA bound to the plasmid. Lane 1: Control plasmid without PNA binding site and reacted with fluorescein-PNA1 and rhodamine-PNA2. Lane 2: Plasmid containing both the PNABS1 and PNABS2 binding sites and labeled with fluorescein-PNA1. Lane 3: Plasmid containing both the PNABS1 and PNABS2 binding sites and labeled with rhodamine-PNA2. Lane 4: Same plasmid labeled with both fluorescein-PNA1 and rhodamine-PNA2. The bands containing the PNABS1 and PNABS2 binding sites are shown by the arrows. 
ed cells. Gold-labeled plasmid DNA can be seen in the extracellular space (Figure 3C, top) and in the cytoplasm (Figure 3C, top, middle and bottom). It was also observed attached to the cell surface and in endocytotic vesicles
(Figure 3C, middle and bottom).

The coupling of peptides or oligonucleotides to plasmid with the PNA-biotin-streptavidin linkage was accomplished by using the heterobifunctional cross-linker SMCC, which reacts with streptavidin to form a stable amide bond, leaving a maleimide moiety accessible for reaction with sulfhydryl groups. The peptide used for these studies was an SV40 NLS peptide containing an N-terminal cysteine residue. To detect the
A

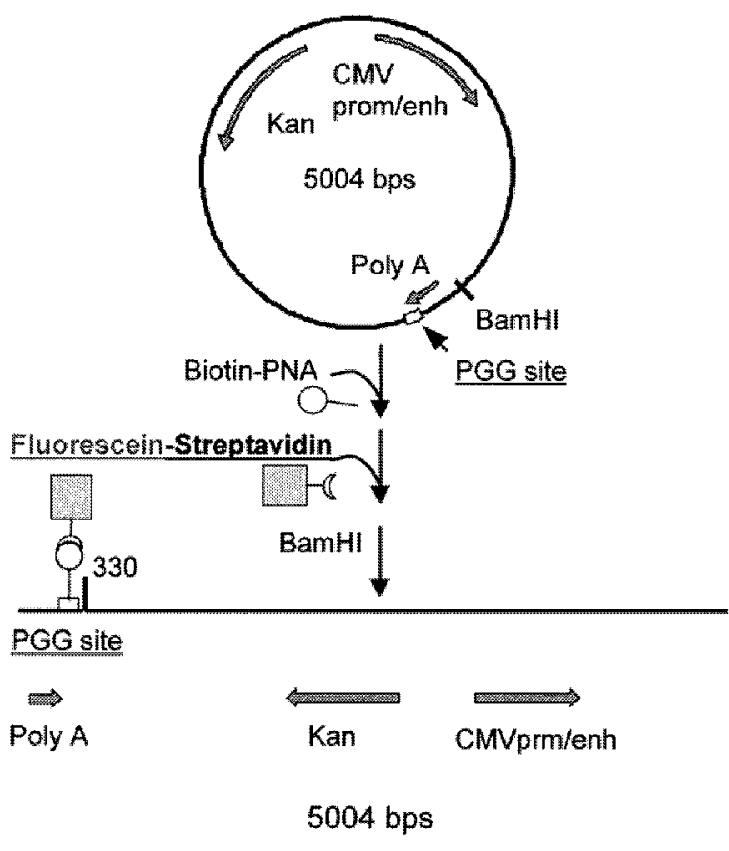

B

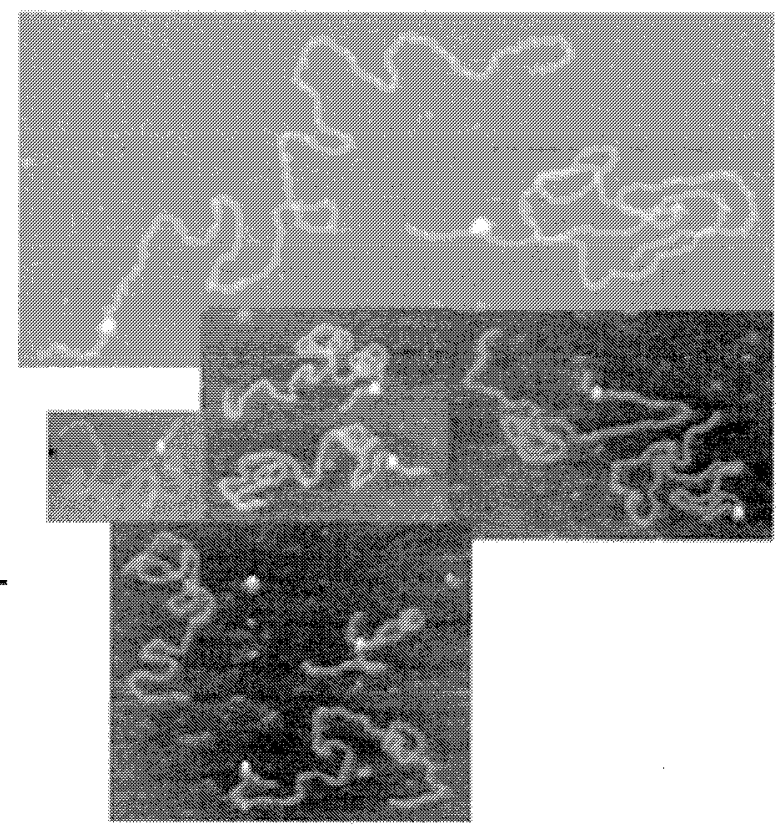

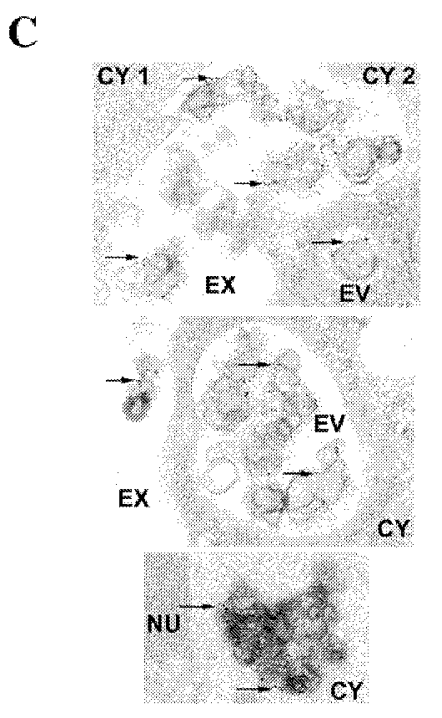

Figure 3. Characterization of streptavidin conjugates binding to biotin-PNA labeled plasmid DNA. (A) Preparation of streptavidin-labeled linearized plasmid DNA. First, biotin-PNA clamp was hybridized to the supercoiled plasmid, then fluorescein-streptavidin was bound to the plasmid/PNA-biotin, and finally the DNA was linearized with BamH1. (B) Cryo-AFM of linearized, biotin-PNA-labeled plasmid containing bound streptavidin. The white spot at the end of each strand shows the location of the bound streptavidin. (C) Electron microscope pictures of COS 7 cells transfected $48 \mathrm{~h}$ earlier with streptavidin-gold-labeled plasmid DNA. The arrows show the gold-labeled DNA. Gold-labeled DNA is seen in the extracellular space attached to the cell plasma membrane and inside the cells. CY1: Cytoplasm of cells No. 1; CY2: cytoplasm of cells No. 2; EX: extracellular space; EV: endocytotic vesicles; and NU: nucleus. 
peptide, it was first labeled with the fluorescent succinimidyl Alexa 488 dye that reacts with the primary amino groups (lysine residues) on the peptide. After being reduced with DTT, the fluorescent peptide was reacted with streptavidinSMCC, and the reaction mixture was passed over a Sephadex G-50 column to produce the purified streptavidin-NLSAlexa conjugate. The Streptavidin-conjugated oligonucleotide was prepared in a similar way. The oligonucleotide used for these studies was a thiol-modified, fluorescein-labeled oligonucleotide containing immunostimulatory $\mathrm{CpG}$ elements (18). After reduction of $\mathrm{CpG}$ ODN by DTT, the free sulfhydryl group was reacted with streptavidin-SMCC, and the resulting streptavidin conjugate was purified by Sephadex G-50 column chromatography.

The streptavidin-NLS-labeled plasmid was prepared by adding the purified streptavidin-NLS to biotin-PNAlabeled plasmid, and the product was analyzed by agarose gel electrophoresis. The results in Figure 4A show that the fluorescent-NLS-streptavidin conjugate was bound to the biotin-labeled plasmid (Figure 4A, lane 3). The conjugate did not bind to the same plasmid labeled with PNA-SMCC (Figure 4A, lane 4) showing that streptavidin binding to the plasmid was dependent on the presence of biotin-PNA on the plasmid, as expected.

Streptavidin-CpG ODN-labeled plasmid was prepared by adding the purified streptavidin-CpG ODN conjugate to biotin-PNA-labeled plasmid. The labeled DNA was similarly analyzed by agarose gel electrophoresis, and the results are shown in Figure 4B. The fluoresceinCpG ODN-streptavidin conjugate bound to biotin-labeled plasmid (Figure 4B, lane 3) but not to SMCC-PNA-labeled plasmid (Figure 4B, lane 4). These results show that sulfhydryl-containing peptides and oligonucleotides can be coupled to streptavidin maleimide, and the resulting streptavidin conjugates bind to biotin-PNA-labeled plasmid.

\section{Direct Use of PNA-SMCC to Link Ligands to Plasmid DNA}

Another PDGC approach is illustrated in Figure 5. Here, the maleimide moiety is conjugated directly to the
PNA. The NHS ester end of the SMCC was first reacted with the $5^{\prime}$ primary amine of the PNA clamp to form a stable amide bond. Then, the maleimidePNA conjugate was hybridized to its binding site on the plasmid. The results in Figure 5, A and B, show that peptides or oligonucleotides containing a free sulfhydryl group can react directly with the maleimide moiety on the
PNA-DNA hybrid. Plasmid DNA containing the PNA binding site was incubated with SMCC-PNA to allow hybridization and the mixture was ethanol precipitated to remove free PNA.

The NLS peptide containing a terminal cysteine residue was reduced and mixed with maleimide-PNA labeled plasmid. The mixture was purified by ethanol precipitation and examined by

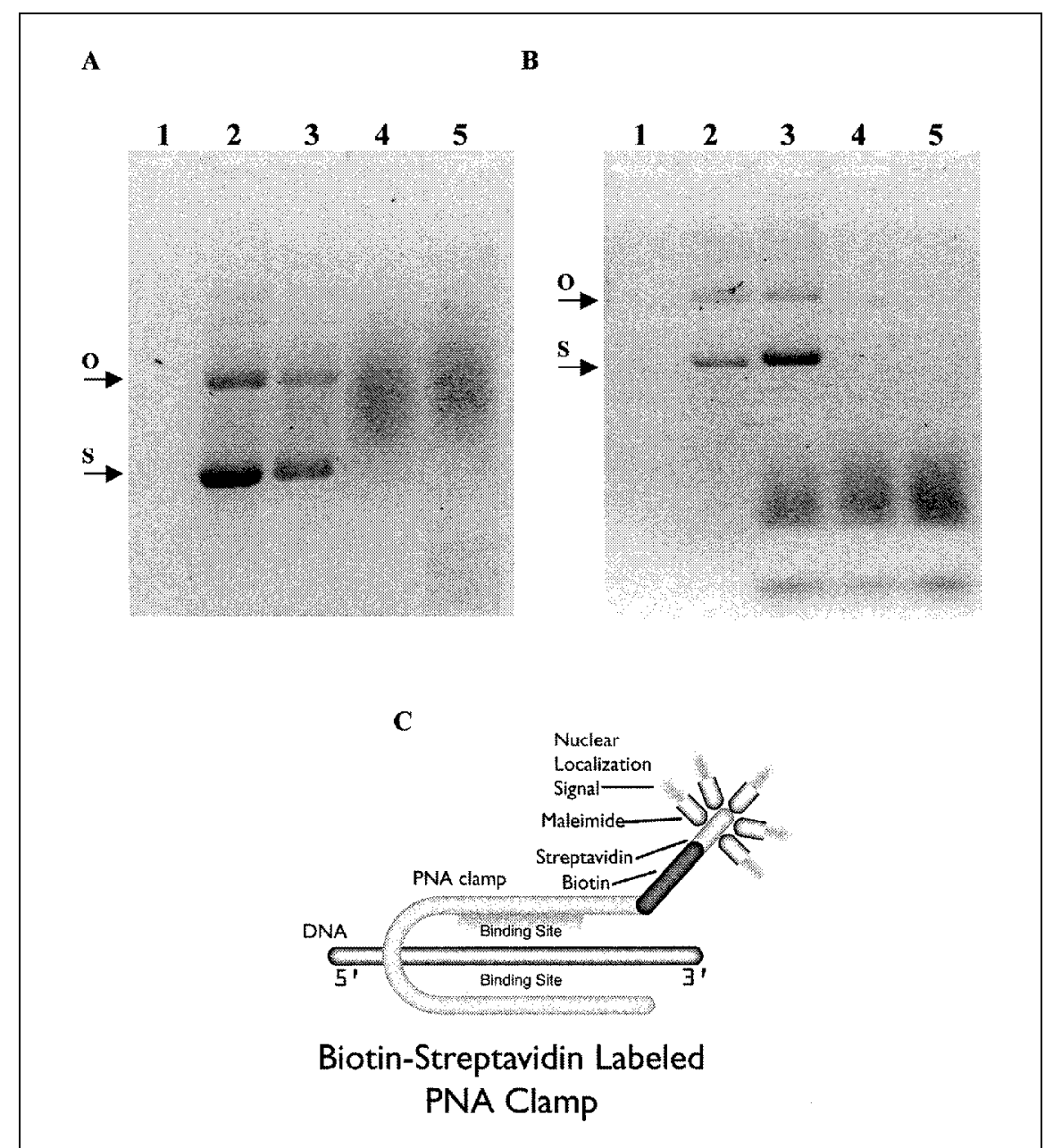

Figure 4. Coupling of an NLS peptide and a CpG ODN to plasmid using the streptavidin-biotinPNA linker and analyzed by agarose gel electrophoresis. (A) lane 1: biotin-PNA-labeled plasmid; lane 2: biotin-PNA-labeled plasmid incubated with Alexa-labeled streptavidin. Plasmid/PNA-biotinstreptavidin-Alexa; lane 3: biotin-PNA-labeled plasmid incubated with Alexa-NLS-conjugated streptavidin. Plasmid/PNA-biotin-streptavidin-NLS peptide-Alexa; lane 4: SMCC-PNA-labeled plasmid incubated with the Alexa-NLS-conjugated streptavidin; and lane 5: the Alexa-NLS streptavidin conjugate alone. (B) lane 1: biotin-PNA-labeled plasmid; lane 2: biotin-PNA-labeled plasmid incubated with fluorescein-streptavidin. Plasmid/PNA-biotin-streptavidin-FITC; lane 3: biotin-PNA-labeled plasmid incubated with fluorescein-ODN-conjugated streptavidin. Plasmid/PNA-biotin-streptavidin-CpG ODN-fluorescein; lane 4: SMCC-PNA labeled plasmid incubated with fluorescein-ODN-conjugated streptavidin; and lane 5: the fluorescein-ODN streptavidin conjugate alone. (O: Open circular plasmid. S: supercoiled plasmid.) (C) Illustration of the use of biotin-PNA and streptavidin to couple peptides, proteins and oligonucleotides to DNA. The plasmid/PNA-biotin hybrid is used to capture streptavidin. A heterobifunctional cross-linker can be used to covalently attach peptides, proteins, oligonucleotides or other types of ligand to streptavidin. When these streptavidin-ligand conjugates are added directly to biotin-PNA-labeled plasmid, they will bind irreversibly to the biotin-PNA plasmid hybrid. 
agarose gel electrophoresis. The results in Figure 5A show that the plasmid containing reactive maleimide became labeled with the NLS (lane 2), but a plasmid containing biotin-PNA was not labeled (lane 3). These results demonstrate that labeling is dependent on the reaction between the reduced sulfhydryl group and maleimide moiety on the PNA. DNA/PNA-fluorescein was used as a control to show where the DNA migrated into the gel (Figure 5A, lane 5). The results in Figure 5B show that the reaction also works with the thiolated CpG ODN. The CpG fluorescent ODN bound to DNA when SMCC-PNA was hybridized to the plasmid (lane 2), but not when biotinPNA was used (lane 3). Other controls showed that $\mathrm{CpG}$ fluorescein-labeled ODN alone migrated to the bottom of the gel (lane 5) and the fluoresceinPNA-labeled DNA shows that the plasmid migrated to the location of the supercoiled and open circular plasmid forms (lane 6).

\section{$\mathbf{A}$}

\section{B}

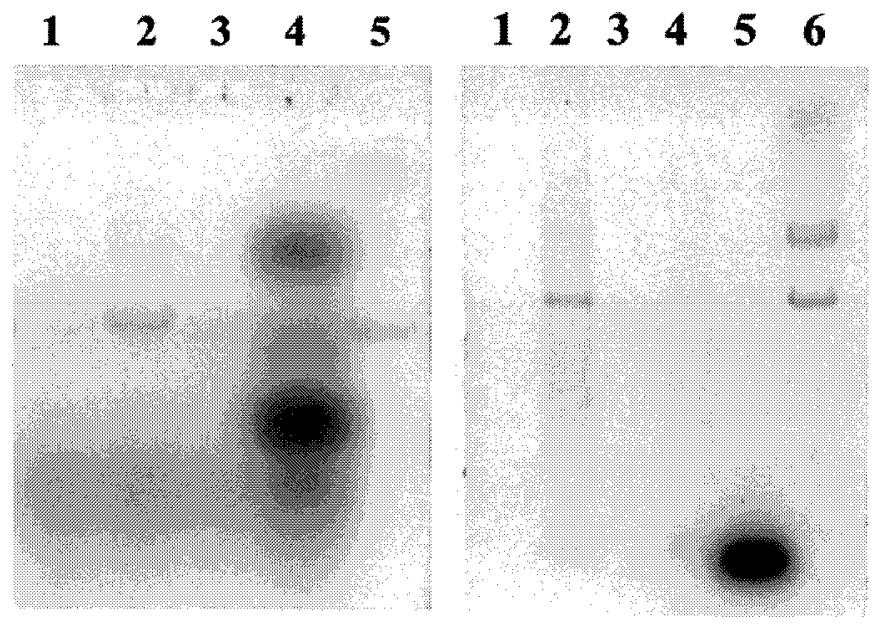

C

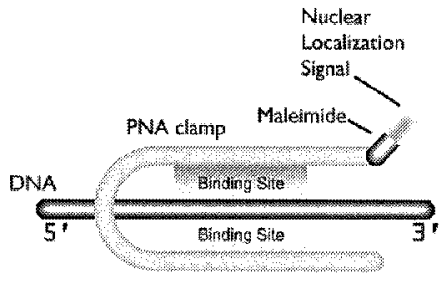

Maleimide Labeled

PNA Clamp

Figure 5. Coupling of a NLS peptide and a CpG ODN to plasmid using the SMCC-PNA linker analyzed by agarose gel electrophoresis. (A) lane 1: SMCC-PNA-labeled plasmid; lane 2: NLS peptide conjugated to SMCC-PNA-labeled plasmid. Plasmid/PNA-SMCC-NLS peptide; lane 3: biotin-PNA-labeled plasmid incubated with the NLS peptide; lane 4: NLS peptide alone; and lane 5: control plasmid labeled with a fluorescein-PNA. Plasmid/PNA-fluorescein. NLS peptide was visualized after coupling with the ATTO-TAG labeling kit, which reacts with the primary amines present on the NLS peptide. (B) lane 1: SMCC-PNA-labeled plasmid; lane 2: Fluorescein-ODN conjugated to SMCC-PNA-labeled plasmid. DNA/PNA-SMCC-ODN-fluorescein; lane 3: biotin-PNA-labeled plasmid; lane 4: biotin-PNA-labeled plasmid incubated with the fluorescein-ODN; lane 5: fluorescein-ODN alone; and lane 6: control plasmid labeled with a fluorescein-PNA. DNA/PNA-fluorescein. (C) Illustration of the use of maleimide-PNA to couple peptides, proteins and oligonucleotides to DNA. An SMCC-PNA clamp is hybridized to plasmid DNA. Peptides (such as an NLS), proteins, oligonucleotides or other types of ligands containing sulfhydryl groups can be mixed with the SMCC-PNA/DNA hybrid to form a chemically stable product. 


\section{DISCUSSION}

In a previous report, we described how fluorophore-conjugated PNAs could be used to produce fluorescently labeled plasmid by hybridizing the fluorescent PNA to a binding site cloned into the plasmid $(16,22)$. This labeling method does not change the supercoiled conformation of the plasmid or its ability to be efficiently transcribed. We now show how this method can be used to couple peptides and oligonucleotides onto DNA and describe two approaches. The first approach involves making peptide- or oligonucleotide-streptavidin conjugates and then binding them to biotin-PNA-labeled plasmid. The streptavidin conjugates are prepared by reacting streptavidin-maleimide with ligands containing a free thiol residue. The second approach uses a maleimide-PNA conjugate, which can be hybridized to the plasmid and then reacted with a ligand containing a free thiol residue.

The PDGC technology that is described in this report will give investigators a means of carrying out medicinal chemistry with genes. Medicinal chemistry is a classical approach to drug discovery that is commonly used for small molecule drug development to make systematic chemical modifications of biologically active agents to improve their bioavailability and efficacy. Being able to carry out a medicinal chemistry approach to improve the bioavailability and efficacy of DNA is presently lacking because the methods that have been used to directly modify DNA either reduce or destroy its ability to be transcribed. Since PNA binding is sequence dependent, it can be designed to bind to a region of the plasmid that does not interfere with the promoter/enhancer or the coding region and so it enables chemistry to be carried out on the PNA/DNA hybrid without destroying the activity of the gene.

Binding of peptides or proteins to plasmids may influence their delivery and expression in ways that are illustrated in Figure 6. A ligand (PNA-lig) that binds to a cell surface receptor can be attached to a plasmid, directing the plasmid specifically to the targeted cell. An endosomolytic peptide (PNA-lyt) can be attached to the DNA so that endocytosed plasmid can more efficiently escape the endosomal compartment. An NLS peptide (PNA-nuc) attached to the plasmid may enable it to pass more efficiently across the nuclear membrane of nondividing cells. By using different PNA binding sites together with their complementary PNA clamps, different ligands can be attached to the same plasmid at the same time. This strategy will eventually enable the assembly of DNA containing a targeting ligand, an endosomolytic element and an NLS all attached to the same plasmid. Naked DNA is al-

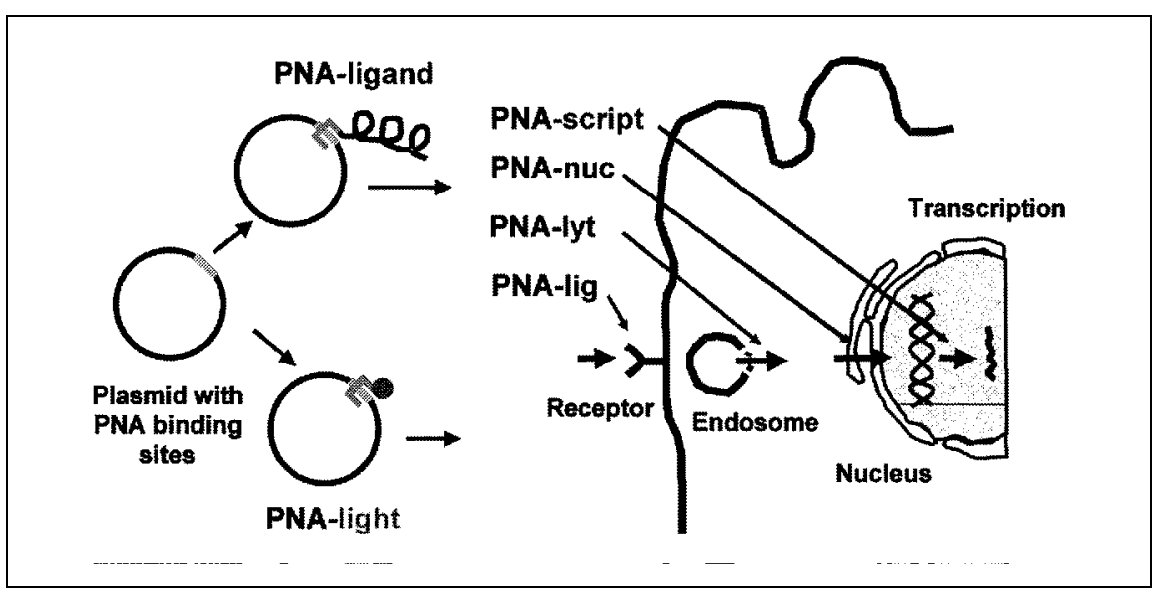

Figure 6. Schematic illustration of the different potential applications of the PDGC technology. A ligand specifically targeting a cell surface receptor can be attached to a plasmid (PNA-lig). An endosomolytic peptide (PNA-lyt) can be attached to the DNA to facilitate cytoplasmic release of the plasmid. A NLS peptide (PNA-nuc) linked to the plasmid may improve plasmid nuclear import. A transcription factor peptide (PNA-script) linked to the plasmid by the PNA clamp could allow transcription without involving intracellular transcription factors. A fluorophore attached to the PNA (PNA-light) can be used to simultaneously monitor both the plasmid DNA biodistribution and the expression of its encoded protein. ready active in many in vivo settings; the addition of one or more of these elements might be expected to improve its in vivo delivery and expression.

In addition to improving plasmid delivery, PDGC has other potential applications that are not directly related to gene therapy. With respect to DNA vaccines, we show that oligonucleotides containing immunostimulatory sequences can be attached to plasmid. Oligonucleotides of this kind may be used to improve the immunogenicity and vaccine potency of DNA vaccines (18). In the virology field, it will be possible to test for the activity of individual viral proteins by binding them to plasmids; transfecting plasmids containing viral proteins into cells may influence their delivery or expression. In the area of signal transduction, a transcription activation peptide (PNAscript in Figure 6) may allow the plasmid to be transcribed without requiring intracellular transcription factors. In this way, transcription factors or peptides may be directly studied for their ability to modulate transcription.

Two approaches are described here for linking peptides and oligonucleotides to plasmids. In the first case, the ligands are bound to the plasmid through a streptavidin bridge, and the ligands are displayed in the context of a protein environment. In the second case, the ligands are coupled directly to the PNA clamp and are presented in close apposition to the DNA. The microenvironments in the immediate vicinity of peptides that are displayed in these two contexts are quite different, and so their biological activities may also differ. For example, a positively charged nuclear localization peptide that is coupled directly to DNA through the PNA clamp might be expected to interact strongly with the negatively charged DNA phosphodiester backbone, which makes it inaccessible to the proteins of the nuclear pore complex. But nuclear localization signals bound directly to proteins, such as BSA and streptavidin, have already been shown to retain their ability to deliver the conjugated protein into the nucleus of cells $(1,14)$. Thus, when examining the effect of a ligand on the delivery or expression of a plasmid, it will be important to compare the activities of the 
ligand as displayed in both the protein (streptavidin) environment and in closer apposition to the DNA without the streptavidin bridge.

The PDGC technology has been designed to be extremely versatile. The copy number of a ligand bound to the plasmid can be varied according to the need, simply by cloning into the plasmid the desired number of PNA binding sites. In addition, we have also shown that different PNA binding sites can be used to incorporate different ligands into the same plasmid. For example, a nuclear localization peptide can be added to one of the sites and a fluorophore to the second site. In this way, the changes in the intracellular biodistribution of the plasmid mediated by the NLS can be conveniently monitored. A plasmid containing three different PNA binding sites could be constructed with a ligand for a cell surface receptor, an endosomolytic peptide and an NLS all on the same plasmid. Therefore, the application of this technology will provide a versatile tool to help elucidate biological mechanisms in cell biology and physiology and ultimately, to help improve the performance of synthetic gene delivery systems for applications in gene therapy.

\section{ACKNOWLEDGMENTS}

Financial support for this work was provided by National Institutes of Health Grant Nos. 1R44CA80598 and RR07720 and National Science Foundation Grant No. DBI9730060.

\section{REFERENCES}

1.Adam, S.A., R.S. Marr and L. Gerace. 1990. Nuclear protein import in permeabilized mammalian cells requires soluble cytoplasmic factors. J. Cell Biol. 111:807-816.

2.Bukanov, N.O., V.V. Demidov, P.E. Nielsen and M.D. Frank-Kamenetskii. 1998. PDloop: a complex of duplex DNA with an oligonucleotide. Proc. Natl. Acad. Sci. USA 95:5516-5520.

3.Cherny, D.Y., B.P. Belotserkovskii, M.D. Frank-Kamenetskii, M. Egholm, O. Buchardt, R.H. Berg and P. Nielsen. 1993. DNA unwinding upon strand-displacement binding of a thymine-substituted polyamide to double-stranded DNA. Proc. Natl. Acad. Sci. USA 90:1667-1670.

4.Demidov, V.V., M.V. Yavnilovich, B.P. Belotserkovskii, M.D. Frank-Kamenetskii and P. Nielsen. 1995. Kinetics and mechanism of polyamide ("peptide") nucleic acid binding to duplex DNA. Proc. Natl. Acad. Sci. USA 92:2637-2641.

5.Donnelly, J.J., J.B. Ulmer, J.W. Shiver and M.A. Liu. 1997. DNA vaccines. Annu. Rev. Immunol. 15:617-648.

6.Egholm, M., O. Buchardt, L. Christensen, C. Behrens, S.M. Freier, D.A. Driver, R.H. Berg, S.K. Kim, B. Norden and P. Nielsen. 1993. PNA hybridizes to complementary oligonucleotides obeying the Watson-Crick hydrogen-bonding rules. Nature 365:566-568.

7.Egholm, M., O. Buchardt, P. Nielsen and R.H. Berg. 1992. Peptide Nucleic Acids (PNA). Oligonucleotide analogues with an achiral peptide backbone. J. Am. Chem. Soc. 114:1895-1897.

8.Egholm, M., L. Christensen, K.L. Dueholm, O. Buchardt, J. Coull and P.E. Nielsen. 1995. Efficient pH-independent sequence-specific DNA binding by pseudoisocytosine-containing bis-PNA. Nucleic Acids Res. 23:217222.

9.Felgner, J.H., R. Kumar, C.N. Sridhar, C.J. Wheeler, Y.J. Tsai, R. Border, P. Ramsey, M. Martin and P.L. Felgner. 1994. Enhanced gene delivery and mechanism studies with a novel series of cationic lipid formulations. J. Biol. Chem. 269:2550-2561.

10.Felgner, P.L. 1996. Improvements in cationic liposomes for in vivo gene transfer. Hum. Gene Ther. 7:1791-1793.

11.Felgner, P.L. 1998. DNA vaccines. Curr. Biol. 8:R551-553.

12.Gao, X. and L. Huang. 1995. Cationic liposome-mediated gene transfer. Gene Ther. 2:710-722.

13.Griffith, M.C., L.M. Risen, M.J. Greig, E.A. Lesnik, K.G. Sprankle, R.H. Griffey, J.S. Kiely and S.M. Freier. 1995. Single and bis peptide nucleic acids as triplexing agents: binding and stoichiometry. J. Am. Chem. Soc. 117:831-832.

14.Holaska, J.M. and B.M. Paschal. 1998. A cytosolic activity distinct from Crm1 mediates nuclear export of protein kinase inhibitor in permeabilized cells. Proc. Natl. Acad. Sci. USA 95:14739-14744.

15.Huang, L., M.-C. Hung and E. Wagner. 1999. Non Viral Vectors for Gene Therapy. Academic Press, San Diego.

16.Liang, X., O. Zelphati, C. Nguyen and P.L. Felgner. 1999. Plasmid labeling using PNA, Chapter 3.3. In P.E. Nielsen and M. Egholm (Eds.), Peptide Nucleic Acids: Protocols and Applications. Horizon Scientific Press, Norfolk, England, UK.

17.Nielsen, P., M. Egholm, R.H. Berg and O. Buchardt. 1991. Sequence-selective recognition of DNA by strand displacement with a thymidine-substituted polyamide. Science 254:1497-1500.

18.Sato, Y., M. Roman, H. Tighe, D. Lee, M. Corr, M.D. Nguyen, G.J. Silverman, M. Lotz, D.A. Carson and E. Raz. 1996. Immunostimulatory DNA sequences necessary for effective intradermal gene immunization. Science 273:352-354.

19.Sheng, S. and Z. Shao. 1998. Biological Cryo-Atomic Force Microscopy: Instrumentation and Applications. Jpn. J. Appl. Phys. 37:3828-3833.

20.Xu, Y. and F.C. Szoka, Jr. 1996. Mechanism of DNA release from cationic liposome/DNA complexes used in cell transfection. Biochemistry 35:5616-5623.

21.Zabner, J., A.J. Fasbender, T. Moninger, K.A. Poellinger and M.J. Welsh. 1995. Cellular and molecular barriers to gene transfer by a cationic lipid. J. Biol. Chem. 270:1899719007.

22.Zelphati, O., X. Liang, P. Hobart and P.L. Felgner. 1999. Gene chemistry: functionally and conformationally intact fluorescent plasmid DNA. Human Gene Ther. 10:15-24.

23.Zelphati, O. and F.C. Szoka, Jr. 1996. Mechanism of oligonucleotide release from cationic liposomes. Proc. Natl. Acad. Sci. USA 93:11493-11498.

Received 27 August 1999; accepted 12 October 1999.

Address correspondence to:

Dr. Philip L. Felgner

Gene Therapy Systems

10190 Telesis Court

San Diego, CA 92121, USA

Internet:pfelgner@genetherapysystems.com 